\title{
Thirty years of colposcopic studies: Validity of local destructive treatments Panama 1982-2012
}

\author{
J orge Luis Garrido \\ CHEMSA Clinic, Panama city, Panama. \\ Correspondence: Jorge Luis Garrido. Address: Justo Arosemena Ave., 33 E St., Kalenkeris Building, Ground floor, \\ Panama city, Panama. Email: dr.jlgarrido0405@gmail.com
}

Received: January 17, 2014

DOI : $10.5430 /$ jnep.v4n8p140

\begin{abstract}
Since our intention was to verify the effectiveness of local destructive treatments which were applied to patients with oncogenic risk due to their cervical pathology, we followed 396 patients during 30 years. These treatments were applied almost immediately after the initial diagnosis was made.

The evaluated parameters were the pathology type, the type of treatment, patients' evolution, and the Polymerase Chain Reaction (PCR) results. The latter is a molecular biology test which was applied to 119 cases between 2011 and 2012, this was introduced as a type of quality control and a way to confirm whether patients were actually healed or improved. With regards to the PCR results, even if the patient was considered to be cured or improved, only 5 of 119 cases were negative, and among those that resulted positive, most of them were HR-HPV, being these 31, 18, 35 and 16. The final result of the study indicates $82 \%$ of the patients were healed; $8.3 \%$ persisted with their condition; $4.8 \%$ improved; $2.8 \%$ recurred and only one case progressed, being this only $0.2 \%$.
\end{abstract}

\section{Key words}

Oncogenic risk, Diagnosis, Treatment, Evolution

\section{I ntroduction}

In the 30 years of this study 12,679 patients were treated, and 6,411 of them were diagnosed as oncogenic risk. Of the latter group 5,498 corresponded to non-clinical HPV or clinical HPV associated with dysplasia or cancer. The providers guided most of these patients towards local destructive treatments (LDT). A follow up was performed to evaluate the effectiveness of that treatment, either cryotherapy, or cauterization; all of the patients accepted treatment types mentioned in the medical literature ${ }^{[1]}$.

We realized that achieving a natural immune response against HPV infection is almost impossible, since the acquired immune response requires an inflammatory reaction. This observation doesn't quite agree with what Machado states in his article: that HPV develops both a natural and induced immune response in the cervical stroma, yet he also states it is unpredictable ${ }^{[3]}$. According to our results, with cryotherapy and/or cauterization we managed to activate the immune system. This is demonstrated by routine colposcopy, applied every three months until clinical cure is achieved, that is to say, the destruction of viral cytopathic effect, visible to colposcopy. This was the same methodology applied in all 6 
provinces we visited over the past 30 years, and the same method applied in the study performed by the Dicle University between $2000-2009^{[2,4,5]}$.

When we talk about cryotherapy, we mean that we provoke a lesion in the affected area by freezing it at approximately $-60^{\circ} \mathrm{C}$, killing the cells by water intoxication, while cauterization produces desiccation of cells and thus, the expected immunological, local, regional and systemic response. The preferred method is cryotherapy, in which we first freeze the lesion over 2 to 3 minutes; then it is slowly defrosted; then the lesion is frozen again for two minutes maximum, and it is slowly defrosted again. The preferred method was applied, either by itself or combined, obtaining good results.

The treatment is complemented by using tetracycline, vitamin A and Albothyl. Within a month, we conducted clinical inspection and then, after three months a routine colposcopy is performed.

\section{Material and method}

We selected 396 patients with O.R. diagnosis, who had their follow ups over the past 30 years, corresponding to 265 HPV cases, 113 dysplasia cases, 7 cancer cases and 11 pure O.R. cases (see Table 1).

Table 1. Cases of oncogenic risk under surveillance

\begin{tabular}{lll}
\hline O.R. classification & $\mathbf{N}^{\circ}$ of cases & Percentage \\
\hline HPV & 265 & $66.9 \%$ \\
Dysplasia & 113 & $28.5 \%$ \\
Cancer & 7 & $1.8 \%$ \\
Pure O.R. & 11 & $2.8 \%$ \\
Total & 396 & $100 \%$ \\
\hline
\end{tabular}

Among the group, we used cryotherapy alone 321 times; a combination of cryotherapy and cauterization was used 51 times; cauterization alone was only used 87 times. The treatments were repeated as often as necessary, if the clinical lesion could not be eradicated in one or three months.

The treatment was done as soon as we knew about the oncogenic risk diagnosis. A follow-up was done after three months, and a new treatment was applied, if the lesion persisted. After the lesion was removed, successive controls were done every six months and/or annually.

Patients were assisted with clinical controls every 6 months or every year, achieving a definitive diagnosis, in which healing prevailed. Once the PCR test was applied after $1-5$ years in 199 cases, 10 years in 94 cases, 15 years in 41 cases, 20 years in 25 cases and 30 years with 12 cases, all results were positive, except for five negative cases; all of the results were HR-HPV (see Table 2).

Table 2. Elapsed time and PCR

\begin{tabular}{ll}
\hline Periods & $\mathbf{N}^{\circ}$ of patients \\
\hline 1 - 5 years & 199 \\
6 - 10 years & 94 \\
11 - 15 years & 41 \\
16 - 20 years & 25 \\
21 - 30 years & 12 \\
Negative & 5 \\
Pending results & 20 \\
Total & 396 \\
\hline
\end{tabular}




\section{Results}

From the 396 cases, 326 of them were considered cured due to the elimination of the viral cytopathic effect, verified by routine colposcopy. Only 33 cases persisted, 19 improved, 11 recurred and only one case progressed (among dysplasia cases). Within dysplasia cases, there were 2 pending cases, and among HPV, 4 pending cases.

From a pathological classification perspective, healing was achieved in 100\% of cancer cases and pure Oncogenic Risk (O.R.), but dysplasia cases and pure HPV cases prevailed with 98 and 210 cured cases respectively, followed by persistence with 31 cases and improvement with 12 cases (see Table 3, 4 and 5).

Table 3. PCR and pathologic evolution

\begin{tabular}{lllllll}
\hline Pathology & Healing & Persistence & Improvement & Recurrence & Progression & TOTAL \\
\hline Pure O.R. & 11 & 0 & 0 & 0 & 0 & 11 \\
Cancer & 7 & 0 & 0 & 0 & 0 & 7 \\
Dysplasia & 98 & 2 & 12 & 3 & 1 & 111 \\
HPV & 210 & 31 & 19 & 11 & 0 & 261 \\
Total & 326 & 33 & & & 1 & $390 *$ \\
\hline
\end{tabular}

*6 pending cases for clinical assessment

Table 4. Clinical evolution by pathologic diagnosis

\begin{tabular}{lllll}
\hline \multirow{2}{*}{ Clinical evolution } & \multicolumn{2}{l}{ Pathology } & & \\
\cline { 2 - 5 } & Cancer (\%) & Pure O.R. (\%) & Dysplasia (\%) & HPV (\%) \\
\hline Healing & 100 & 100 & 88.2 & 80.4 \\
Persistence & - & - & 1.8 & 11.9 \\
Improvement & - & - & 6.3 & 4.6 \\
Recurrence & - & - & 2.7 & 3.1 \\
Progression & - & - & 0.9 & 100 \\
Pending & - & - & $\sim 100$ & \\
\hline
\end{tabular}

Table 5. Clinical evolutions

\begin{tabular}{lll}
\hline Clinical evolution & No. patients & Percentage \\
\hline Healing & 326 & $82 \%$ \\
Persistence & 33 & $8.3 \%$ \\
Improvement & 19 & $4.8 \%$ \\
Recurrence & 11 & $2.8 \%$ \\
Progression & 1 & $0.2 \%$ \\
Pending & 6 & $1.5 \%$ \\
Total & 396 & $100 \%$ \\
\hline
\end{tabular}

Since healing was the prevailing clinical presentation, the intention was to apply the PCR test as a quality control. The PCR test was applied to 119 women, and our findings indicate that only 5 cases were negative and the rest were considered as HR-HPV with the most frequently found subtypes 31, 18, 35 and 16 subtypes the most frequently found, with 40, 27, 19 and 17 cases respectively (see Table 6).

These results with the healing clinical findings occurred, despite the virus remaining in the human body, without producing any cytological or histologic changes that might lead to cancer (as a host) over the last 30 years is what allow us to say that the local destructive treatment has achieved on one hand, the HPV lesion destruction, and, on the other, its 
neutralization by specific immunology. However what we cannot endorse is that the HPV chronic infection leads to, which is cancer (see Table 6).

Table 6. PCR in 119 cases

\begin{tabular}{lll}
\hline PCR results & No. patients & Percentage (\%) \\
\hline 7 & 2 & 1.7 \\
16 & 17 & 14.2 \\
18 & 27 & 22.7 \\
31 & 40 & 33.7 \\
35 & 19 & 16.0 \\
66 & 1 & 0.8 \\
Negative & 5 & 4.2 \\
Others & 8 & 6.7 \\
Total & 119 & 100 \\
\hline
\end{tabular}

\section{Discussion}

Some important questions arise: Why is it that it takes so long for a patient to be diagnosed in the early stages of the disease (e.g. HPV infection or dysplasia)? Why is it that the patients usually are treated when the disease has advanced, and furthermore, with such aggressive treatments? What did we do differently that allowed us to have such rates of success among our patients? One of the reasons could be the use of routine colposcopy in all of our patients. Monsonego mentions the latest guidelines regarding the indications of colposcopy. This test is still indicated after abnormal cytological results, especially ASC-H, LSIL, HSIL and AGC. It is also indicated in the women with ASC-US who have positive results to high risk HPV (HR-HPV). When cytology is combined with HPV - DNA testing in women over 30 years old, colposcopy is indicated when HR-HPV is detected twice in a 9 months period ${ }^{[6]}$. Nevertheless, there are some other authors, such as Bozse, who mention colposcopy as part of a routine gynecological examination, with its advantages and disadvantages ${ }^{[7]}$.

In this study, we opted for the routine colposcopy as we have done in other studies ${ }^{[8]}$. This allowed us to confirm the impact of routine colposcopy on the diagnosis and prognosis of our patients, by letting us treat the patients on time and in a conservative way, thus, obtaining excellent results. Nevertheless, even when the patients seem to be clinically cured, PCR results indicate otherwise. Now the question is: why is that the PCR test turns out positive even when the patients were treated and proven to be cured by colposcopy, or haven't presented any sign or symptom of oncogenic risk by the time of the study? It is hard to obtain a clear significance of these results. Besides, logic tells us that we should obtain negative results from most of the treated patients. The presence of a pathological PCR in real time in those patients that were strictly following their health control regimen every 6 months and who were considered healthy after they were examined can suggest:

1) A balanced immune response against the HPV and its oncogenic potential

2) A stigma left behind after the HPV infection was treated.

3) Both

In this research, the treatment of choice is cryotherapy. One of the main reasons why we choose cryotherapy over other treatments (e.g. cauterization or topical treatment) is because the $\mathrm{CO}_{2}$ allows us to reach temperatures such as $-60^{\circ} \mathrm{C}$, and with that the destruction of both healthy and infected cells, which could generate a personal or specific immune response and with this, we can justify the treated virus inactivity. This cryotherapy is combined with topical and oral treatments: tetracycline, Vitamin A and Albothyl, which has proven to be more effective than cryotherapy alone. If we compare it versus the cases treated with cauterization only, healing reached lower rates, which is why we complement it with 
chemical treatments, achieving in most cases healing, that is, the immune personal response, that the virus itself won't provide.

In the end, this management has allowed us to evaluate the therapeutic method, individual response, PCR linkage, evolutional diagnosis, and over all, send clear concepts to patients in order for the patients to have a better quality of life.

\section{Conclusions}

In these 30 years of studies, we have demonstrated that local conservative treatments are valid for three simple reasons:

1) The costs of aggressive treatments, compared with surgical ones, are much more expensive.

2) The risk, to which the patient is exposed with aggressive treatments, sometimes causes significant damage to the organs.

3) It makes no sense that a low-risk diagnosis must be reached by applying aggressive treatments.

Our experience with the patients' management, indicate to us that their clinical evolution is succesful, since there is no progression to invasive cancer.

\section{References}

[1] Dexeus S.: “CIN Treatments.” Eur. J. Gynaecol. Oncol. 1989; 20(3): 215.

[2] Garrido J.L.: “Screening cervical cancer 27 years experience in six Rep. of Panama.” Clin. Exp. Obst. \& Gynecol. 2012; 39(3): 343. PMid:23157040

[3] Machado F.A., Jenssen J.P., Michelin M.A., Murta E.F.: "Immune response and immunotherapy in intraepithelial and invasive lesion of uterine cervix.” Clin. Exp. Obstet. Gynecol. 2012; 39(1): 27. PMid:22675951

[4] Delingeoroglou E., Christopulos P., Aravantinos L., Papadias K.: "Human papilloma virus molecular profile and mechanism of cancerogenesis: a review.” Eur. J. Oncol. Gynaecol. 2009; 30(2): 128.

[5] Kuyumcuoglu U., Hocaoglu S., Guzel A.I., Celik Y. “The clinical significance of HPV screening in premalignant cervical lesions.” Eur. J. Oncol. Gynaecol. 2010; 31(5): 596.

[6] Monsonego J. "Colposcopy: the value of HPV testing in clinical practice”. Gynecol., Obstet. \& Fertil. 2004; 32(1): 62-74. PMid:14736603 http://dx.doi.org/10.1016/j.gyobfe.2003.10.025

[7] Bozse P. “Colposcopy used in primary setting (routine colposcopy): advantages and concerns.” Eur. J. Oncol. Gynaecol. 2006; 27(1): 5.

[8] Garrido Martínez J.L., Diaz M.M., Villarreal A.: “Twenty years of contributions to the study and prevention of uterine cancer” Eur J. Gynaecol. Oncol. 2006; 27(6): 600-602. PMid:17290591 